\title{
Principais Causas de Dermatite de Contacto Alérgica Ocupacional: Um Estudo de Três Anos no Centro de Portugal
}

\section{Main Causes of Occupational Allergic Contact Dermatitis: A Three Year Study in the Center of Portugal}

Catarina PESTANA $\bigotimes^{1}$, Raquel GOMES ${ }^{2}$, Vítor PINHEIRO ${ }^{1}$, Miguel GOUVEIA ${ }^{3}$, Isabel ANTUNES ${ }^{1}$, Margarida GONÇALO $^{3,4}$ Acta Med Port 2016 Jul-Aug;29(7-8):449-455 - http://dx.doi.org/10.20344/amp.6605

\section{RESUMO}

Introdução: A dermatite de contacto alérgica, tal como a dermatite de contacto irritativa e as reações imediatas, urticária de contacto, são as principais doenças dermatológicas profissionais mas raramente notificadas aos sistemas nacionais de vigilância de riscos profissionais. Pretendemos avaliar as profissões mais frequentemente relacionadas com o diagnóstico de dermatite de contacto alérgica, os alergénios mais frequentemente envolvidos e a sua relação com a modificação da exposição profissional.

Material e Métodos: Efetuámos um estudo retrospetivo (2012 - 2014) nos pacientes que realizaram testes epicutâneos na Consulta de Alergologia do Serviço de Dermatologia do Centro Hospitalar e Universitário de Coimbra. Foram estudadas as características demográficas dos pacientes com dermatite de contacto alérgica ocupacional, identificados os alergénios, caracterizada a sua profissão e o efeito da modificação ou abandono do posto de trabalho na evolução da doença.

Resultados: Entre os 941 doentes testados, 77 (8,2\%) sofriam de dermatite de contacto alérgica ocupacional comprovada por 169 testes epicutâneos positivos relacionados com exposição laboral, 55 detetados na série básica e 114 nas séries complementares de alergénios. A maior parte dos casos envolvia as mãos $(88,3 \%)$ e as atividades profissionais mais afetadas foram os cabeleireiros/estética de unhas devido à manipulação de (met)acrilatos, os alergénios mais frequentemente encontrados entre as dermatites de contacto alérgicas ocupacionais. Destes doentes $27,3 \%$ abandonaram o trabalho, 23,4\% mudaram de posto de trabalho e $49 \%$ fizeram evicção do alergénio responsável, do que resultou a resolução da dermatite de contacto alérgica em $39 \%$ dos casos e melhoria noutros $39 \%$, não havendo qualquer melhoria das lesões cutâneas em $22 \%$.

Discussão: Este estudo, apesar de incluir apenas doentes da zona centro do país, avalia um número elevado de doentes com profissões variadas e testados com extensas séries de alergénios. Os alergénios e profissões classicamente referidas (mistura de tiurans, parafenilenodiamina, crómio, cobalto e em profissionais de saúde, cabeleireiros e construção civil) diagnosticados pela série básica de alergénios foram largamente ultrapassados pelos (met)acrilatos, a principal causa de dermatite de contacto alérgica ocupacional, particularmente em esteticistas. Salientamos ainda, de forma transversal a várias profissões, a metilsiotiazolinona como o segundo alérgeno mais frequente, certamente relacionado com a corrente 'epidemia' de alergia de contacto a este conservante.

Conclusão: Apesar de a estética ungueal não ser referida como uma profissão de elevado risco de dermatite de contacto alérgica na maioria dos estudos, as tendências atuais da moda com o recurso frequente a unhas de gel, o desempenho desta profissão por indivíduos habitualmente pouco informados quanto aos riscos, aliado ao elevado potencial sensibilizante dos (met)acrilatos, motiva certamente a elevada frequência destes casos entre nós.

Palavras-chave: Alergénios; Dermatite de Contacto Alérgica; Dermatite Ocupacional; Metacrilatos; Testes epicutâneos.

\section{ABSTRACT}

Introduction: Allergic contact dermatitis, along with irritant contact dermatitis and immediate contact reactions, contact urticarial, are the most frequent dermatological occupational disease, but seldom reported to the National authorities.

Material and Methods: We performed a 3-year retrospective study at the allergology section in the Dermatology Clinic of the University Hospital of Coimbra to evaluate the main occupations diagnosed as occupational allergic contact dermatitis, most common allergens and the effect of the modification of the work station in the evolution of the disease.

Results: During 2012 - 2014 among the 941 patch tested patients, 77 (8.2\%) were diagnosed with occupational allergic contact dermatitis, with 169 positive patch tests related to occupational exposure, 55 detected within the baseline and 114 in complementary test series. In most cases allergic contact dermatitis involved the hands (88.3\%), main professional activities were nail estheticians and hairdressers due to the manipulation of (meth)acrylates, the most common allergen in the study. After the diagnosis, $27.3 \%$ abandoned the work, $23.4 \%$ changed the work station, $49 \%$ avoided exposure to the responsible allergen. Contact dermatitis resolved in $39 \%$ of the patients, improved in $39 \%$ but had no change in the remaining $22 \%$.

Discussion: This study, although including only patients from the center of Portugal, evaluates a large sample of patients with different occupations studied with a larger variety of allergens. Apart from classical allergens and professions responsible for occupational allergic contact dermatitis that we found in lower numbers (thiuram mix, paraphenylenodiamine, chromium and cobalt in health care workers, hairdressers and in the building industry), (meth)acrylates tested outside the European and Portuguese Baseline Series were the main cause of occupational allergic contact dermatitis, namely in nail estheticians. Methylisothiazolinone, the second more frequent occupational contact allergen in the present study was identified in different occupations as a result of the widespread use of this preservative that is causing a real 'epidemics' of allergic contact dermatitis all over Europe in the last years.

1. Serviço de Saúde Ocupacional. Centro Hospitalar e Universitário de Coimbra. Coimbra. Portugal.

2. Serviço de Imuno-alergologia. Centro Hospitalar e Universitário de Coimbra. Coimbra. Portugal.

3. Serviço de Dermatologia. Centro Hospitalar e Universitário de Coimbra. Coimbra. Portugal.

4. Clinica de Dermatologia. Faculdade de Medicina. Universidade de Coimbra. Coimbra. Portugal.

$\square$ Autor correspondente: Catarina Pestana. catarinapestana28@gmail.com

Recebido: 12 de maio de 2015 - Aceite: 08 de janeiro de 2016 | Copyright @ Ordem dos Médicos 2016 
Conclusion: Nail estheticians are not usually referred as an occupation with a high risk of developing allergic contact dermatitis. Nevertheless, the current fashion combined with professionals poorly informed about the risk of their activity and the high sensitizing potential of (meth)acrylates, leads to a higher frequency of allergic contact dermatitis in recent years.

Keywords: Allergens; Dermatitis, Allergic Contact/etiology; Dermatitis, Occupational/etiology; Methacrylates; Patch Tests; Portugal.

\section{INTRODUÇÃO}

As dermatites de contacto, que incluem as formas irritativas, alérgicas e as reações imediatas, representam a principal doença dermatológica profissional. ${ }^{1-3}$

A dermatite de contacto alérgica (DCA) é uma reação de hipersensibilidade retardada (tipo IV) mediada por linfócitos T específicos do alergénio, habitualmente uma substância química de baixo peso molecular a que o indivíduo está exposto. Necessita de sensibilização prévia que depende do potencial sensibilizante da substância química, da forma e dose de exposição e, ainda, de outros fatores favorecedores como a coexistência de fatores irritantes (detergentes, solventes, pós), alterações da barreira epidérmica e fatores individuais e genéticos. ${ }^{1-3}$

Uma vez sensibilizado, as ulteriores exposições à mesma substância ou a outras quimicamente aparentadas podem desencadear, em 12 - 48 horas, prurido, pápulas, vesículas e exsudação, ou nas formas de exposição crónica descamação, liquenificação e/ou fissuração. Estas apresentações clínicas do espectro de eczema dependem da substância causal, do local e forma de exposição (aguda ou, mais frequentemente, crónica e repetida). ${ }^{1-3}$

A DCA ocupacional afeta sobretudo as mãos, por vezes com extensão aos punhos, antebraços e outras áreas expostas, como a face e pescoço. Para o diagnóstico de DCA ocupacional, além da observação e história clínica, em particular da relação da dermatose com as atividades desempenhadas no local de trabalho, os testes epicutâneos (patch test) são o método diagnóstico mais importante para a identificação do(s) alergénio(s) causais. Ainda que considerada frequente, entre nós a DCA ocupacional é raramente notificada às instituições oficiais que regulamentam o risco de doenças profissionais (no ano de 2008 apenas foram reportadas 12 doenças dermatológicas ocupacionais). ${ }^{4}$

O presente estudo teve por objetivo determinar a frequência de DCA de causa ocupacional entre os doentes que foram submetidos a testes epicutâneos num período de três anos numa clínica da região centro do país e avaliar as profissões e alergénios mais frequentemente envolvidos. Foi também avaliado o efeito do abandono do posto de trabalho e/ou evicção dos alergénios responsáveis na evolução da doença.

\section{MATERIAL E MÉTODOS}

Foi efetuado um estudo retrospetivo durante um período compreendido entre janeiro de 2012 e dezembro de 2014 com os doentes que efetuaram testes epicutâneos na Consulta de Alergologia Cutânea, no serviço de Dermatologia do Centro Hospitalar e Universitário de Coimbra (Portugal) para estudo de suspeita de DCA ou outra reação de hipersensibilidade retardada com impacto cutâneo.

Foram considerados apenas os doentes com testes epicutâneos positivos a alergénios presentes nos seus locais de trabalho e cuja exposição contribuía de forma significativa para desencadear ou agravar a dermatite. Foram avaliados os seguintes parâmetros: género, idade, história pessoal de atopia, localização das lesões e necessidade de recurso a terapia sistémica, como forma de traduzir a gravidade da patologia, tempo de evolução das lesões, profissão e tempo na profissão até ao início da dermatite, séries testadas, alergénios positivos e se houve modificação no posto de trabalho (abandono completo ou apenas evicção ou redução da exposição ao alergénio) e resultado subsequente.

Os alergénios foram aplicados no dorso em Finn Chambers $^{\circledR}$ on Scanpor ${ }^{\circledR}$ Tape (Almirall Hermal GmbH, Germany) ou em câmaras IQ-Ultra ${ }^{\mathrm{TM}}$ (Chemotechnique Diagnostics $^{\mathrm{TM}}$, Vellinge, Sweden) e retirados às $48 \mathrm{~h}$. Em todos os doentes foi aplicada a série básica europeia e portuguesa do GPEDC (Grupo Português de Estudo das Dermatites de Contacto) e séries complementares de alergénios, escolhidas com base na história de exposição ocupacional ou outra (Trolab, Almirall Hermal GmbH, Germany ou Chemotechnique Diagnostics ${ }^{\mathrm{TM}}$, Vellinge, Sweden). Efetuaram-se por vezes testes epicutâneos ou testes abertos com produtos trazidos pelo doente, recolhidos no local de trabalho ou no seu ambiente pessoal. A leitura dos testes foi realizada ao segundo ou terceiro dia (D2/D3) e quarto ou sétimo dia (D4/D7), segundo as recomendações do International Contact Dermatitis Research Group e da European Society of Contact Dermatitis (ESCD). ${ }^{5}$ As reações positivas foram interpretadas como tendo relevância atual, passada, desconhecida ou reação cruzada.

Para a análise estatística dos resultados foi utilizado o teste não paramétrico Qui-quadrado, com a utilização do programa IBM SPSS Statistics para Windows, versão 22.0.

\section{RESULTADOS}

Do total de 941 doentes testados no período de três anos ( $251-26,7 \%$ do sexo masculino; $690-73,3 \%$ do sexo feminino) foram selecionados $77(8,2 \%)$ que cumpriam os critérios de diagnóstico de DCA ocupacional, com média de idades de $38,1 \pm 11,9$ anos, $62(80,5 \%)$ dos quais do sexo feminino. Foi encontrada história pessoal de atopia em $16(20,8 \%)$, rinite alérgica em sete $(9 \%)$, asma em seis $(7,8 \%)$ e eczema atópico em três $(3,9 \%)$.

Dezoito doentes $(23,4 \%)$ sofriam de DCA grave com necessidade de terapêutica sistémica, eczema que afetava a face e pescoço em $9(11,7 \%)$ e as mãos em $68(88,3 \%)$, nestes casos com destaque para dermatite das polpas digitais e da zona peri-ungueal.

Entre as 77 profissões, predominam as esteticistas $(n=24 ; 31,2 \%)$ e cabeleireiras $(n=5 ; 6,5 \%)$, algumas 
Tabela 1 - Profissões dos pacientes com testes epicutâneos positivos

\begin{tabular}{lc}
\hline Profissões & Atuais \\
\hline Cabeleireira/ esteticista & 32 \\
Esteticista & 24 \\
Cabeleireira & 5 \\
Ambas & 3 \\
Profissionais de saúde & 14 \\
Enfermeiros & 7 \\
Auxiliares de ação médica & 3 \\
Técnica de estomatologia/ auxiliar consultório dentário & 3 \\
Médico & 1 \\
Construção civil & 9 \\
Cozinheira & $\mathbf{8}$ \\
Indústria de metalomecânica e automóvel & $\mathbf{5}$ \\
Indústria de vitrocerâmica & 3 \\
Agricultora e profissões relacionadas & $\mathbf{3}$ \\
Animador de festas criança/ educadora de infância & $\mathbf{2}$ \\
Fábrica de móveis & $\mathbf{3}$ \\
\hline Total & $\mathbf{7 7}$ \\
\hline
\end{tabular}

acumulando as duas atividades $(n=3 ; 3,9 \%)$ ou realizando a atividade de esteticista em part-time $(n=1 ; 1,3 \%)$, os trabalhadores da área da saúde $(n=14 ; 18,2 \%)$, da construção civil ( $n=9 ; 11,7 \%$ ) e os cozinheiros ( $n=8 ; 10,4 \%$ ) (Tabela 1). Dezasseis doentes $(20,8 \%)$ estavam na profissão há menos de um ano e $53 \%$ há menos de cinco.

As séries complementares de alergénios mais frequentemente testadas foram as de conservantes e veículos de cosméticos e produtos de higiene pessoal (43 doentes; $55,8 \%$ ), de (met)acrilatos $(n=33 ; 42,9 \%)$, de aditivos da borracha/luvas ( $n=33 ; 42,9 \%)$, cabeleireiros ( $n=11$; $14,3 \%$ ), plantas ( $n=9 ; 11,7 \%$ ) e o dissulfureto de dialilo e alimentos (t.q.) ( $n=8 ; 10,4 \%)$. Testes abertos foram realizados em 22 doentes (28,6\%), 60\% dos quais com produtos utilizados no posto de trabalho.

Encontraram-se 169 reações positivas a alergénios a que atribuímos relevância ocupacional ainda que alguns possam coexistir no ambiente doméstico, como por exemplo as isotiazolinonas, metilisotiazolinona (MI) e clorometilisotiazolinona (MCl), ${ }^{6,7}$ e o níquel (Tabela 2). Cinquenta e cinco reações positivas foram observadas na série básica de alergénios enquanto que 114 alergénios (67,5\%) foram identificados nas séries complementares. Considerando apenas a série básica, encontrámos uma associação positiva estatisticamente significativa entre DCA ocupacional e alguns alergénios, nomeadamente a metilisotiazolinona, mistura de tiurans, crómio, formaldeído e resina epoxídica, enquanto que para o níquel e a colofónia se verificou uma associação negativa (Tabela 2).
No total, os principais alergénios foram o hidroxietilmetacrilato (HEMA) ( $\mathrm{n}=30 ; 17,8 \%)$, hidroxipropilmetacrilato (HPMA) $(n=26 ; 15,4 \%)$, a metilisotiazolinona ( $n=12$; $7,1 \%$ ), a mistura de tiurans ( $n=9 ; 5,3 \%$ ) e o dissulfureto dialilo ( $n=7 ; 4,1 \%$ ) (Tabelas 2 e 3 ).

A maioria dos 77 casos foi relacionada com a utilização de (met)acrilatos ( $n=32 ; 42 \%)$ em estética de unhas $(n=$ $27 ; 35 \%$ ) e em estomatologia ( $n=3$, auxiliares em consultório dentário e de prótese dentária). Quatro esteticistas tiveram também testes positivos à metilisotiazolinona presente em produtos de higiene utilizados no local de trabalho e no ambiente doméstico.

Das oito cabeleireiras $(10,4 \%$ da amostra), seis (75\%) tiveram teste positivo para a parafenilenodiamina (PPD) presente nas tintas capilares, cinco $(62,5 \%)$ à metilisotiazolinona e três $(37,5 \%)$ ao formaldeído, conservantes presentes em champôs, no caso do último alergénio sob a forma de libertadores de formaldeído.

Dos nove trabalhadores da construção civil e de estradas $(11,7 \%$ da amostra), sete $(77,7 \%)$ tiveram sensibilização ao cobalto e/ou crómio (constituintes do cimento e das luvas de proteção em couro) e cinco $(55,6 \%)$ reagiram às misturas de tiurans e/ou carbamatos das luvas de borracha (Tabela 3).

Das oito cozinheiras observadas, sete tinham sensibilização ao dissulfureto dialilo (alergénio do alho), duas à cenoura e uma à cebola.

Dos 14 profissionais de saúde $(18,2 \%)$, assistentes operacionais, enfermeiros e médicos, dois tiveram testes positivos à mistura de tiurans das luvas de proteção, duas enfermeiras demonstraram sensibilização a antibióticos do grupo das cefalosporinas que preparam diariamente e um médico oftalmologista reagiu a anestésicos locais com que contactava durante cirurgias e observação de doentes. Observámos três testes abertos compatíveis com reação alérgica a Softaskin ${ }^{\circledR}$ gel de limpeza e desinfeção das mãos utilizado em unidades de saúde. Contudo, não identificámos o alergénio responsável e não foi possível eliminar completamente um possível efeito irritativo desta preparação no teste aberto realizado.

Os três trabalhadores da área da agricultura/estufas de plantas foram positivos à mistura de lactonas sesquiterpénicas e extratos de plantas que as contêm (Chamomila romana, Tanacetum vulgare, óleo de louro e parthenolide) e, ainda, a alfa-metileno-gama-butirolactona (tulipalina) da Alstroemeria.

Num animador de festas de crianças observou-se DCA aos aditivos da borracha dos balões (mistura de tiurans), uma educadora de infância tinha sensibilização à lanolina, ao perfume lyral (hidroximetilpentilciclohexano-carboxaldeído) e ao conservante metilisotiazolinona, ambos presentes nos cremes que aplicava e nas toalhitas e gel de limpeza que usava diariamente no cuidado dos bebés.

Encontramos ainda sensibilização à resina epoxida e seus constituintes (2-monometilol-fenol, epicloridrina e bisfenol A) em dois trabalhadores da fábrica de turbinas eólicas, ao cobalto em dois trabalhadores da indústria 
Tabela 2 - Comparação dos testes positivos a alergénios da Série básica nos pacientes com e sem DCA ocupacional e causa da DCA nos casos ocupacionais

\begin{tabular}{|c|c|c|c|c|c|}
\hline $\begin{array}{l}\text { Alergénios reativos na Série } \\
\text { Básica }\end{array}$ & $\begin{array}{l}\text { Testes }+ \text { no total } \\
\text { de pacientes } \\
(n=941) \\
n(\%)\end{array}$ & $\begin{array}{c}\text { Testes }+ \text { em } \\
\text { pacientes sem } \\
\text { DCA ocupacional } \\
(n=864) \\
n(\%)\end{array}$ & $\begin{array}{c}\text { Testes }+ \text { em } \\
\text { pacientes com } \\
\text { DCA ocupacional } \\
(\mathrm{n}=77) \\
\mathrm{n}(\%)\end{array}$ & $\begin{array}{l}\text { Associação } \\
\text { de DCA } \\
\text { ocupacional e } \\
\text { alergénios }\end{array}$ & $\begin{array}{l}\text { Causa da DCA } \\
\text { ocupacional }\end{array}$ \\
\hline $\begin{array}{l}\text { MI (500 ppm água) e/ou } \\
\text { MCI/MI (100 ppm água) }\end{array}$ & $71(7,5 \%)$ & $59(6,8 \%)$ & $12(15,6 \%)$ & $\begin{array}{l}\text { Positiva, } \\
p<0,005\end{array}$ & $\begin{array}{l}\text { Champô, produtos } \\
\text { de higiene pessoal/ } \\
\text { trabalho }\end{array}$ \\
\hline Mistura de tiurans $1 \%$ vas & $26(2,8 \%)$ & $17(2,0 \%)$ & $9(11,7 \%)$ & $\begin{array}{l}\text { Positiva, } \\
p<0,001\end{array}$ & \multirow[t]{2}{*}{ Luvas de proteção } \\
\hline Mistura de carbamatos $3 \%$ vas & $13(1,4 \%)$ & $11(1,3 \%)$ & $2(2,6 \%)$ & n.s. & \\
\hline Dicromato de potássio $0,5 \%$ vas & $26(2,8 \%)$ & $21(2,4 \%)$ & $5(6,5 \%)$ & $\begin{array}{l}\text { Positiva, } \\
p=0,039\end{array}$ & $\begin{array}{c}\text { Cimento/luvas de } \\
\text { couro }\end{array}$ \\
\hline Cloreto de cobalto $1 \%$ vas & $61(6,5 \%)$ & $56(6,5 \%)$ & $5(6,5 \%)$ & n.s. & Cimento \\
\hline Parafenilenodiamina $1 \%$ vas & $51(5,4 \%)$ & $46(5,3 \%)$ & $5(6,5 \%)$ & n.s. & \multirow{2}{*}{ Tintas capilares } \\
\hline Disperso laranja $1 \%$ vas & $25(2,7 \%)$ & $23(2,7 \%)$ & $2(2,6 \%)$ & n.s. & \\
\hline Formaldeído $1 \%$ água & $11(1,2 \%)$ & $8(0,9 \%)$ & $3(3,9 \%)$ & $\begin{array}{l}\text { Positiva, } \\
p=0,02\end{array}$ & Champôs \\
\hline Resina epóxida $1 \%$ vas & $7(0,7 \%)$ & $4(0,5 \%)$ & $3(3,9 \%)$ & $\begin{array}{l}\text { Positiva, } \\
p=0,02\end{array}$ & $\begin{array}{c}\text { Colas de contacto, } \\
\text { tintas das turbinas } \\
\text { eólicas }\end{array}$ \\
\hline Sulfato de níquel $5 \%$ vas & $244(25,9 \%)$ & $241(27,9 \%)$ & $3(3,9 \%)$ & $\begin{array}{l}\text { Negativa, } \\
p<0,01\end{array}$ & $\begin{array}{c}\text { Indústria } \\
\text { metalomecânica }\end{array}$ \\
\hline Colofónia $20 \%$ vas & $19(2,0 \%)$ & $18(2,1 \%)$ & $1(1,3 \%)$ & $\begin{array}{l}\text { Negativa, } \\
p<0,01\end{array}$ & Cêra depilatória \\
\hline Alcooís da lanolina $30 \%$ vas & $39(4,1 \%)$ & $37(4,3 \%)$ & $2(2,6 \%)$ & n.s. & Cremes de proteção \\
\hline Isopropil-PPD $0,1 \%$ vas & $13(1,4 \%)$ & $12(1,4 \%)$ & $1(1,3 \%)$ & n.s. & $\begin{array}{l}\text { Borracha negra de } \\
\text { manípulo }\end{array}$ \\
\hline Lyral (HICC) $5 \%$ vas & $14(1,5 \%)$ & $13(1,5 \%)$ & $1(1,3 \%)$ & n.s. & Toalhetes limpeza \\
\hline Mistura de lactonas $0,1 \%$ vas & $10(1,1 \%)$ & $9(1,0 \%)$ & $1(1,3 \%)$ & n.s. & Plantas \\
\hline
\end{tabular}

n.s.: Não significativo; MI: Metilisotiazolinoa; MCl: Metilcloroisotiazolinona.

vitrocerâmica, ao níquel e paládio num trabalhador de uma fábrica de torneiras, a constituintes da borracha negra (isopropil-PPD e ciclofenil-PPD) na indústria automóvel, ao acrilato de butilo numa fábrica de candeeiros e à difenilguanida e lanolina num trabalhador de uma fábrica de móveis.

Dos 77 doentes, $21(27,3 \%)$ abandonaram o trabalho devido à dermatose profissional, 18 (23,4\%) mudaram de posto de trabalho dentro da mesma atividade e 38 (49\%) tentaram a evicção do alergénio responsável. Houve resolução da dermatite em 30 (39\%) e melhoria noutros 30 (39\%), mas sem qualquer modificação em 17 (22\%), todos no último grupo que apenas tentaram a evicção do alergénio. Apenas oito destes casos foram notificados ao Centro Nacional de Proteção contra os Riscos Profissionais.

\section{DISCUSSÃO}

As publicações nacionais relacionadas com DCA ocupacional são escassas e habitualmente com envolvimento de pequenas amostras. ${ }^{8-11}$ Este estudo, ao abarcar um período de três anos, abrange uma amostra mais ampla - não só pela variedade de alergénios como pelas profissões en- volvidas - mas não traduz a realidade nacional, visto que inclui apenas pacientes da região centro do país. Tal como em estudos internacionais, a faixa etária mais frequentemente observada foi a dos $20-29$ anos $(32,5 \%)$, sendo que $80 \%$ tinham menos de 50 anos, correspondendo à faixa de maior produtividade da população portuguesa. Nesta amostra verificou-se ainda que a DCA surgiu mais frequentemente nos primeiros anos de atividade profissional. ${ }^{3}$

Neste estudo $20,7 \%$ dos doentes tinham antecedentes de atopia mas apenas uma minoria referia história de eczema atópico, considerado um fator de risco para desenvolvimento de dermatite de contacto, mas sobretudo para a dermatite de contacto irritativa que não foi avaliada neste trabalho. ${ }^{1}$

A DCA foi considerada grave pois obrigou a terapêutica com corticoterapia sistémica em $23 \%$ dos doentes, mas não foram avaliados o número de dias de ausência de trabalho, que representa um custo significativo, nem a interferência da DCA profissional na qualidade de vida do trabalhador, que se sabe ser habitualmente muito elevada no eczema das mãos. ${ }^{12}$ 
Tabela 3 - Alergénios positivos nas séries complementares e com produtos trazidos pelo paciente e sua relação com DCA ocupacional

\begin{tabular}{|c|c|c|}
\hline Alergénios & $\mathbf{n}$ & Causa \\
\hline \multicolumn{3}{|l|}{ Série de met(acrilatos) $(n=65)$} \\
\hline 2-hidroxietil metacrilato (HEMA) $2 \%$ vas & 30 & Estética ungueal, próteses dentárias \\
\hline 2-hidroxipropil metacrilato (HPMA) $2 \%$ vas & 26 & Estética ungueal, próteses dentárias \\
\hline Trietilenoglicoldimetacrilato (TEGDMA) $2 \%$ vas & 4 & Estética ungueal \\
\hline Etilenoglicol dimetacrilato (EGDMA) $2 \%$ vas & 3 & Estética ungueal \\
\hline Etilacrilato $0,1 \%$ vas & 1 & Estética ungueal \\
\hline 2-Hidroxi-etilacrilato $0,1 \%$ vas & 1 & Estética ungueal \\
\hline \multicolumn{3}{|l|}{ Série de plásticos e colas /óleos de corte $(n=5)$} \\
\hline Benzoisotiazolinona $0,05 \%$ vas & 1 & Luvas \\
\hline 2-Monometilol-fenol $1 \%$ vas & 1 & Resinas, tintas \\
\hline Bisfenol A $1 \%$ vas & 1 & \multirow{2}{*}{ Colas de contacto, tintas } \\
\hline Epicloridina $0,1 \%$ vas & 1 & \\
\hline Acrilato de butilo $0,1 \%$ vas & 1 & Colas \\
\hline \multicolumn{3}{|l|}{ Série de aditivos de borrachas/luvas $(n=8)$} \\
\hline Dissulfureto de tetraetiltiuram (TETD) $0,25 \%$ vas & 2 & \\
\hline Dissulfureto de tetrametiltiuram (TMTD) $0,25 \%$ vas & 1 & Luvas \\
\hline Dipentaetiltiuram (DPT) $0,25 \%$ vas & 1 & \\
\hline $\mathrm{N}, \mathrm{n}$-difenilguanidina $1 \%$ vas & 1 & Borracha utilizada em móveis \\
\hline Diaminodifenilmetano $0,5 \%$ vas & 1 & Tintas \\
\hline Dietil-tioureia $1 \%$ vas & 1 & Luvas \\
\hline Difenil-PPD 1\% vas & 1 & Borracha negra de manípulo \\
\hline \multicolumn{3}{|l|}{ Série de cosméticos/veículos ( $n=2$ ) } \\
\hline Decilglucósido 0,5\% vas & 1 & Champô \\
\hline Resina de tosilamida $10 \%$ vas & 1 & Verniz unhas \\
\hline \multicolumn{3}{|l|}{ Série de plantas $(n=7)$} \\
\hline Alfa-metileno-gama-butirolactona $0,01 \%$ vas & 1 & Alstroemeria \\
\hline Alantolactona $0,033 \%$ & 1 & \\
\hline Chamomilla romana $1 \%$ vas & 1 & Plantas da família das Compostas \\
\hline Tanacetum vulgare extracto $1 \%$ vas & 1 & \\
\hline 8-Metoxipsoraleno $0,01 \%$ vas & $(\mathrm{Ph}) 1$ & \\
\hline Angelica - folha & $(\mathrm{Ph}) 1$ & Plantas que contêm psoralenos \\
\hline Extrato figueira & $(\mathrm{Ph}) 1$ & \\
\hline \multicolumn{3}{|l|}{ Série de cabeleireiras $(n=3)$} \\
\hline p-aminofenol $1 \%$ vas & 1 & Tintas capilares \\
\hline Aminoazobenzeno $0,25 \%$ vas & 1 & Tintas capilares \\
\hline Dietanolamina de coco $0,5 \%$ vas & 1 & Champô \\
\hline \multicolumn{3}{|l|}{ Série de medicamentos tópicos e sistémicos $(n=4)$} \\
\hline Cefotaxima $10 \%$ vas & 2 & Antibióticos \\
\hline Ceftriaxone $10 \%$ vas & 1 & Antibióticos \\
\hline Tetracaína 1\% vas & 1 & Anestésicos locais \\
\hline \multicolumn{3}{|l|}{ Alimentos $(n=10)$} \\
\hline Dissulfureto de dialilo $1 \%$ vas & 7 & Alho \\
\hline Cebola (t.q.) & 1 & Cebola \\
\hline Cenoura (t.q.) & 2 & Cenoura \\
\hline \multicolumn{3}{|l|}{ Produtos trazidos pelos pacientes $(n=6)$} \\
\hline Fragmento de molde de torneira & 1 & Torneira \\
\hline Borracha negra peça de trabalho & 1 & Peça de borracha \\
\hline Massa consistente Isoflex Topas L32 * & 1 & Massa de fechaduras \\
\hline Softaskin gel * & 3 & Gel limpeza \\
\hline Total de reacções positivas relacionadas com a atividade profissional & 114 & \\
\hline
\end{tabular}

\footnotetext{
* Teste aberto; Ph: Teste fotoepicutâneo.
} 
A identificação do agente causal nos testes epicutâneos e a consequente modificação da exposição, com abandono ou mudança de posto de trabalho ou apenas evicção dos alergénios responsáveis pela DCA, permitiu uma melhoria significativa da dermatite na maioria dos doentes observados $(77 \%)$. Salientamos contudo que a série básica, isoladamente não permite diagnosticar mais de dois terços dos casos ocupacionais. É o exemplo da alergia aos (met)acrilatos, a principal causa de DCA ocupacional neste estudo, e que motivou já a inclusão do HEMA na série básica do GPEDC em 2015.

Este grupo de pacientes com DCA ocupacional aos (met)acrilatos (esteticistas) não é habitualmente referido como um dos mais afetados. Porém a larga divulgação da modalidade da estética ungueal ('unhas de gel'), o desconhecimento dos riscos inerentes à manipulação de (met) acrilatos não polimerizados (antes da exposição à radiação ultravioleta), o elevado potencial sensibilizante destes químicos e, neste ambiente profissional, o contacto frequente com superfícies de trabalho contaminadas, a falsa sensação de segurança com o uso de luvas de borracha facilmente permeáveis aos (met)acrilatos e a exposição aos acrilatos volatilizados conjugam-se para facilitar a sensibilização. ${ }^{8}$ A impossibilidade de utilizar as luvas ou dedeiras impermeáveis a (met)acrilatos (luvas $4 \mathrm{H}$ ) nestes trabalhos minuciosos tornam difícil a manutenção da atividade profissional. ${ }^{8}$ Ainda, estes indivíduos sensibilizados deveriam ser informados de outras fontes de exposição a (met)acrilatos, profissionais ou não, pois esta alergia pode condicionar o uso de próteses dentárias ou outro material de correção e limitar algumas atividades profissionais. A ausência de diagnóstico etiológico correto e de informação levou a que uma das pacientes do estudo, após DCA das mãos devido à realização de próteses dentárias, efetuasse um curso de formação em estética ungueal com recidiva das lesões logo com o início desta atividade.

Como profissão frequentemente afetada por DCA ocupacional, os cabeleireiros têm sido alvo de diversos estudos de intervenção bem-sucedidos, minimizando a exposição a alergénios de tintas capilares, líquidos de permanente e champôs, com o ensino do uso adequado de luvas de proteção e a redução dos alergénios nos produtos manipulados, nomeadamente a PPD nas tintas capilares e os tioglicolatos nos líquidos de permanente. Ainda assim, mais de metade das cabeleireiras tiveram teste positivo à PPD e a constituintes de champôs, nomeadamente libertadores de formaldeído (imidazolidinilureia, diazolidinilureia, bronopol ou DMDM-hidantoína) e à $\mathrm{MI}$ ou à mistura $\mathrm{MI} / \mathrm{MCl}^{13-18}$

A MI, presente em cremes de proteção ou produtos de higiene profissional ou pessoal, tem sido responsável por uma epidemia de casos de DCA em Portugal e a nível europeu, e foi também um fator agravante em várias outras profissões neste estudo (enfermeiras, esteticistas, educadoras de infância e cozinheiros) e que se traduziu por uma frequência significativamente superior de reação à MI no grupo de pacientes com DCA profissional (Tabela 2), ${ }^{6,7}$
Ainda que em número inferior a estudos nacionais e internacionais realizados em décadas anteriores, ${ }^{19,20,21}$ salientamos o caso de cinco trabalhadores da construção civil ainda sensibilizados ao crómio do cimento. Atualmente as luvas (pelos aditivos da borracha ou sais de crómio do couro) são as principais causas de DCA nesta profissão, ${ }^{20,21}$ já que a alergia ao crómio do cimento tem vindo a reduzir de forma rápida desde a implementação das diretivas europeias de 2003 que recomendam a adição de sulfato ferroso ao cimento e consequente diminuição do crómio trivalente mais sensibilizante. ${ }^{20-23}$ Contudo, a redução do crómio no material de proteção em couro (sapatos e luvas) não está ainda implementada a nível europeu.

Sendo a região de Coimbra e do centro uma região menos industrializada, é compreensível que as profissões relacionadas com a indústria pesada ocupem uma percentagem relativamente menor entre as DCA ocupacionais encontradas. De igual modo, as medidas de prevenção primária como a automatização da produção em circuitos estanques e a melhoria das condições de trabalho nas grandes unidades poderá justificar este número mais reduzido.

Destes 77 casos de DCA ocupacional apenas oito foram participados ao Centro Nacional de Proteção contra os Riscos Profissionais, o que não permite contribuir para conhecer o panorama de dermatoses ocupacionais entre nós. A notificação pelos profissionais de saúde deveria ser facilitada e incentivada e aos doentes deveriam ser dadas garantias de algum benefício resultante desta participação.

\section{CONCLUSÃO}

A DCA profissional é uma afeção frequente, mas raramente notificada apesar de poder afetar de forma significativa a qualidade de vida e a capacidade produtiva dos trabalhadores. Os testes epicutâneos, com a série básica e com séries complementares adequadas à atividade profissional e com os produtos recolhidos no ambiente profissional, são imprescindíveis para o correto diagnóstico destes doentes, orientam a evicção alergénica e são essenciais para a cura ou melhoria significativa das lesões.

Importa, contudo, referir que a DCA é o resultado de uma sensibilização prévia com expressão para toda a vida do doente pelo que o ideal seria uma prevenção primária (evitar a sensibilização a alergénios no local de trabalho). A entidade patronal e os trabalhadores devem ser alertados para a importância da evicção alergénica e do uso de equipamentos de proteção individual adequados. O material de proteção bem como os produtos de higiene têm um papel ambivalente porque podem também ser causa ou fator de manutenção da doença.

\section{PROTECÇÃO DE PESSOAS E ANIMAIS}

Os autores declaram que os procedimentos seguidos estavam de acordo com os regulamentos estabelecidos pelos responsáveis da Comissão de Investigação Clínica e Ética e de acordo com a Declaração de Helsínquia da Associação Médica Mundial. 


\section{CONFIDENCIALIDADE DOS DADOS}

Os autores declaram ter seguido os protocolos do seu centro de trabalho acerca da publicação de dados.

\section{CONFLITOS DE INTERESSE}

Os autores declaram não ter qualquer conflito de interesse relativamente ao presente artigo.

\section{REFERÊNCIAS}

1. Peng W, Novak N. Pathogenesis of atopic dermatitis. Clin Exp Allergy. 2015;45:566-74.

2. Alchorne A, Alchorne M, Silva M. Occupational dermatosis. An Bras Dermatol. 2010;85:137-47.

3. Johanssen JD, Frosch P, Lepoittevin JP editors. Textbook of contact dermatitis. $5^{\text {th }}$ ed. Berlin: Springer-Verlag; 2011.

4. Jorge A. Avaliação das alterações respiratórias induzidas por exposições ocupacionais através de metodologia não invasiva. [dissertação académica]. Coimbra: Universidade de Coimbra; 2014.

5. Johansen JD, Aalto-Korte K, Agner T, Andersen KE, Bircher A, Bruze M, et al. European Society of Contact Dermatitis guideline for diagnostic patch testing-recommendations on best practice. Contact Dermatitis. 2015;73:196-221.

6. Gameiro A, Coutinho I, Ramos L, Gonçalo M. Methylisothiazolinone: second 'epidemic' of isothiazolinone sensitization. Contact Dermatitis. 2014;70:242-3.

7. Gonçalo M, Goossens A. Whilst Rome burns: the epidemic of contact allergy to methylisothiazolinone. Contact Dermatitis. 2013;68:257-8.

8. Ramos L, Cabral R, Gonçalo M. Allergic contact dermatitis caused by acrylates and methacrylates--a 7-year study. Contact Dermatitis. 2014;71:102-7.

9. Gonçalo S, Figueiredo A, Gonçalo M. Dermatite de contacto profissional pela tetracaína. Boletim Informativo do GPEDC. 1987;1:38.

10. Canelas M, Gonçalo M, Figueiredo A. Contact allergy to epoxy resins-a 10-year study. Contact Dermatitis. 2010;62:55.

11. Antunes J, Silva R, Pacheco D, Travassos R, Filipe P. Occupational contact allergy to cephalosporins. Dermatol Online J. 2011;17:13

12. Agner T, Andersen KE, Brandão FM, Bruynzeel DP, Bruze M, Frosch $P$, et al. Hand eczema severity and quality of life: a cross-sectional, multicentre study of hand eczema patients. Contact Dermatitis. 2008;59:43-7.

13. Lim SW, Smith W, Gillis D, Kette F. IgE-mediated allergy to formaldehyde from topical application. Contact Dermatitis. 2006;54:230.

14. Antelmi A, Young E, Svedman C, Zimerson E, Engfeldt M, Foti C, et

\section{FONTES DE FINANCIAMENTO}

Os autores declaram não ter recebido subsídios ou bolsas para a elaboração do artigo. al. Are gloves sufficiently protective when hairdressers are exposed to permanent hair dyes? An in vivo study. Contact Dermatitis. 2015;72:229 36.

15. Hougaard MG, Winther L, Søsted H, Zachariae C, Johansen JD. Occupational skin diseases in hairdressing apprentices - has anything changed? Contact Dermatitis. 2015;72:40-6.

16. Uter W, Gefeller O, John SM, Schnuch A, Geier J. Contact allergy to ingredients of hair cosmetics - a comparison of female hairdressers and clients based on IVDK 2007-2012 data. Contact Dermatitis. 2014;71:1320.

17. Søsted H, Rustemeyer T, Gonçalo M, Bruze M, Goossens A, GiménezArnau AM, et al. Contact allergy to common ingredients in hair dyes. Contact Dermatitis. 2013;69:32-9.

18. Pontén A, Aalto-Korte K, Agner T, Andersen KE, Giménez-Arnau AM, Gonçalo M, et al. Patch testing with $2.0 \%(0.60 \mathrm{mg} / \mathrm{cm} 2)$ formaldehyde instead of $1.0 \%(0.30 \mathrm{mg} / \mathrm{cm} 2)$ detects significantly more contact allergy. Contact Dermatitis. 2013;68:50-3.

19. Zachariae CO, Agner T, Menné T. Chromium allergy in consecutive patients in a country where ferrous sulfate has been added to cement since 1981. Contact Dermatitis. 1996;35:83-5.

20. Lazzarini R, Duarte IA, Sumita JM, Minnicelli R. Allergic contact dermatitis among construction workers detected in a clinic that did not specialize in occupational dermatitis. An Bras Dermatol. 2012;87:56771.

21. Freitas N, Barros M. Eczema de contacto na construção civil (19552002). Boletim Informativo GPEDC. 2003;17: 20-1.

22. Teixeira V, Coutinho I, Gonçalo M. Allergic contact dermatitis to metals over a 20-year period in the centre of Portugal: evaluation of the effects of the European directives. Acta Med Port. 2014:27:295-303.

23. Parlamento Europeu e do Conselho. I Relatório sobre a proposta de diretiva do Parlamento Europeu e do Conselho relativa à limitação da colocação no mercado e da utilização de nonilfenol, etoxilato de nonilfenol e cimento (vigésima sexta alteração da Diretiva 76/769/CEE do Conselho, de 27 de julho de 1976). 